\title{
Los robots y la Inteligencia Artificial. Nuevos retos del periodismo
}

\section{Robots and Artificial Intelligence: New Challenges of journalism}

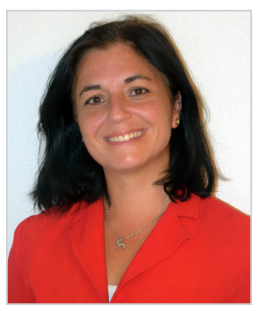

Idoia Salazar. Doctora en Periodismo y profesora en los Grados Internacionales en la Facultad de Comunicación y Humanidades de la Universidad CEU San Pablo. Investigador Principal del Grupo SIMPAIR (Social Impact of Artificial Intelligence and Robotics), especializada en ética en la Inteligencia Artificial. Desarrollo de carrera profesional en los medios digitales del Grupo PRISA durante 17 años, en los que fue responsable en el área de I+D, participando en proyectos competitivos de la Unión Europea, relacionados con tecnologías de web semántica y su implementación en productos del grupo. Autora del libro: Las profundidades de Internet: Accede a la información que los buscadores convencionales no encuentran y descubre el futuro inteligente de la Web, así como de numerosos artículos en medios de comunicación divulgativos. Universidad CEU San Pablo, Madrid, España

idoiaana.salazargarcia@ceu.es

ORCID: 0000-0002-9540-8740

Recibido: 05/06/2018 - Aceptado: 24/09/2018

\section{Resumen:}

Este estudio analiza el destacable impacto social de la Inteligencia Artificial y la Robótica en todos los ámbitos, centrándose en el periodismo. Se recogen distintas iniciativas actuales de desarrollo e implantación de estas nuevas tecnologías en medios de comunicación y se detallan ventajas e inconvenientes de las mismas desde un punto de vista tanto profesional como ético. Asimismo, se han tenido en cuenta diversos estudios, a nivel internacional, sobre el tema tratado y se ha consultado a expertos en la materia sobre la viabilidad real de estas tecnologías en el área que nos ocupa. El resultado permite vislumbrar una nueva forma de hacer periodismo, basada en la colaboración directa hombre-máquina, en la que el periodista deberá redefinirse para ajustarse a la nueva situación.

\section{Palabras clave:}

Inteligencia Artificial; robots; periodismo; tecnología; ética.
Received: 05/06/2018 - Accepted:24/09/2018

\section{Abstract:}

This study analyses the remarkable social impact of Artificial Intelligence (AI) and Robotics in all areas, focusing on journalism. The evolution of these concepts and the influence that science fiction literature and cinematography has had on them is also studied. Different current initiatives of development and implementation of these new technologies in the communication media are collected, and details regarding the advantages and disadvantages of these have been pointed out from a professional and ethical point of view. Likewise, several studies have been taken into account at the international level on the subject dealt with and experts in the field have been consulted on the real viability of these technologies in the area in question. The results allow us to envision a new way of carrying out journalism based on direct human-machine collaboration in which the journalist must redefine him or herself to adjust to the new situation.

\section{Keywords:}

Artificial Intelligence; robots; journalism; Technology; ethics.

Cómo citar este artículo:

Salazar, I. (2018). Los robots y la Inteligencia Artificial. Nuevos retos del periodismo. Doxa Comunicación, 27, pp. 295-315.

https://doi.org/10.31921/doxacom.n27a15 


\section{Introducción}

17 de marzo de 2014. Un terremoto, de 4.4 grados, hace temblar a Los Ángeles (EE.UU). Hasta aquí nada se sale de lo habitual. Pero el mundo del periodismo ese mismo día, y por este mismo hecho iba a sufrir una sacudida propiciada por una reflexión profunda sobre las perspectivas del futuro del periodismo. Y es que el primero en dar esta noticia fue una Inteligencia Artificial (en adelante IA), tan solo tres minutos después de que ocurriera. El medio: El periódico estadounidense Los Ángeles Times. El periodista en cuestión: un algoritmo, creado por el programador Ken Schwencke, capaz de generar artículos cortos. Esta IA se nutre de fuentes fiables y oficiales pre-programadas y no solamente es capaz de publicar noticias de terremotos, sino también sobre el crimen en la ciudad, entre otras temáticas, aunque siempre bajo supervisión y jerarquización de los responsables del diario. Esta simple noticia dio la vuelta al mundo e hizo que, aquellos medios de comunicación que aún no habían apostado por este tipo de tecnologías, comenzaran a planteárselo seriamente.

Es un hecho que la IA y la robótica está provocando una revolución, sin precedentes, que afecta a todos los ámbitos sociales y profesionales. En un futuro próximo este impacto se incrementará a un ritmo exponencial y provocará lo que muchos llaman ya la Cuarta Revolución Industrial. Los robots y la IA ayudarán en la automatización y mejora de muchos de los procesos manuales que hacemos en la actualidad. Pero estas tecnologías traen consigo temores y desconcierto derivados de su rápida aparición y de la desinformación. El campo de la Comunicación no escapará a esta tendencia mundial. Formas de IA ayudarán al comunicador a mejorar procesos y a ofrecer al público unas noticias más personalizadas y ricas en contenidos (Jaemin, 2017).

En primer lugar, antes de empezar a analizar la cuestión, es necesario explicar qué es un algoritmo, esa IA que actualmente está escribiendo noticias. Se trata de un conjunto de reglas que, aplicadas sistemáticamente a unos datos de entrada apropiados, resuelven un problema en un número finito de pasos elementales (Berlanga de Jesús, 2016). De una forma más sencilla se definiría como una serie de instrucciones sencillas que llevan a cabo para resolver un problema. Visto esto cabría plantearse el por qué este algoritmo matemático, aparentemente carente de creatividad y conciencia, está creando este impacto social. Este miedo de la sociedad en general a enfrentarse a este tipo de tecnología. La respuesta hay que buscarla no solo en la ciencia, sino también en la evolución histórica del Ser Humano y en la literatura y cinematografía del siglo XX, calificada como Ciencia-Ficción.

\subsection{La Ciencia Ficción como escenario premonitorio de las nuevas tecnologías actuales}

Los robots no nos son ajenos, incluso los más evolucionados. Han estado presentes en nuestra literatura y cinematografía desde hace tiempo (García, 2014). Estas imágenes, las imaginadas a partir de libros, o las explícitas reproducidas través de la televisión o del cine han provocado que la Sociedad adquiera ciertos prejuicios -tanto negativos, como positivos- a la hora de afrontar los grandes retos de su futuro.

La Ciencia-Ficción formó parte de la cultura del siglo XX. Algunas de sus predicciones irreales ya son posibles, como podría ser el holograma tridimensional de la princesa Leia en La Guerra de las Galaxias. Ahora, en el siglo XXI, la ambición de este tipo de arte escapa incluso a las imaginaciones más ingeniosas. Pero, como veremos a continuación, quien sabe si en un futuro pudieran ser palpables y parte de nuestra rutina diaria. 
Remontémonos a 1917, año en el que el checo Joseph Capek escribió el cuento Opilec en el que ya imaginaba a los primeros autómatas (López Pellisa, 2013). Tres años después, su hermano Karel Capek escribió la obra teatral de Ciencia Ficción Rossum's Universal Robots (RUR) en el que se acuñó, por primera vez, el término robot, derivado de la palabra checa robota, que significa siervo o trabajador (Saiz Lorca, 2002). En ella, la empresa que da nombre a la obra construía humanos artificiales de altas capacidades que asumían las grandes cargas de trabajo de sus trabajadores regulares. Aunque habían sido creados con buenas intenciones, finalmente estallaba la revuelta contra los humanos.

El precedente estaba creado, y su aparente factibilidad llevó a otros autores a seguir esta línea en sus relatos de Ciencia Ficción.

En 1950 el escritor y científico ruso Isaac Asimov, utilizó la palabra "Robótica" en su obra Runaround (Asimov, 1942) y comenzó a hacerse popular a partir de historias breves llamadas I Robot. Su visión, en aquel entonces, preveía las posibles implicaciones éticas de aquellas máquinas que empezaban a tomar forma en la imaginación de los lectores de Asimov. Así, estableció las tres leyes inviolables de la robótica que, hoy día, siguen vigentes en la mente de los científicos que los desarrollan:

- Un robot no puede dañar a un ser humano ni, por inacción, permitir que un ser humano sufra daño.

- Un robot debe cumplir las órdenes de los seres humanos, excepto si dichas órdenes entran en conflicto con la Primera Ley.

- Un robot debe proteger su propia existencia en la medida en que ello no entre en conflicto con la Primera o la Segunda Ley.

Con el tiempo, y al introducir en sus relatos robots cada vez más evolucionados, Asimov completó sus tres leyes con una "Ley Cero", que viene a ser una generalización -o más bien un salto cualitativo- de la Primera Ley, puesto que dice que un robot no puede dañar a la Humanidad ni, por inacción, permitir que la humanidad sufra daño.

Desde entonces, los robots han sido ampliamente representados en la ficción literaria y cinematográfica (González-Jiménez, 2018). La saga de Star Wars nos dejó unos amigables R2 D-2 y C-3PO (Kurtz y Lucas, 1977), siempre dispuestos a ayudar a los Jedi. Su director, George Lucas, les dotó de razonamientos, defectos y virtudes que les asemejaba a los humanos, lo cual creaba cierta simpatía ante la masiva audiencia de estas películas.

Terminator, sin embargo, inundó la mente del espectador de razonables dudas sobre si la evolución de los robots y la inteligencia artificial, en general, será favorable para los humanos. Habrá algunos buenos, como el Terminator (Hurd and Cameron (1984), protagonizado por Arnold Schwarzenegger, y otros incluso más evolucionados y dañinos como el cyborg capaz de cambiar de forma. ¿Cómo luchar contra esto? Esta saga nos presenta un predecible holocausto por dotar de "demasiada inteligencia" a las máquinas. Un dilema más en el que pensar al salir del cine.

Yo robot, inspirado en el libro de Asimov del mismo título (Asimov, 1950) presenta al robot Sonny. La película está ambienta en el año 2035, una época en la que la inteligencia artificial, y todo lo que conlleva, ya forma parte de la realidad cotidiana de la vida en la Tierra. El detective Spooner (Will Smith), que odia a los robots -aunque él mismo reconoce tener implantes robóticos en sus articulaciones, es el encargado de investigar el posible asesinato de un científico a manos de un robot -Sonny-. A pesar de las 3 leyes de la robótica implantadas en los cerebros positrónicos de cada robot, parece que Sonny es 
capaz de evolucionar en sus razonamientos, incluso sobre pasando estas leyes aparentemente inquebrantables. A medida que avanza la película vemos como las máquinas empiezan a razonar por sí mismas, amparadas incluso por sus 3 leyes: VIKI, el cerebro positrónico que dirige a todo este mundo altamente mecanizado, pone en marcha una revolución de los robots, bajo un firme propósito: proteger a la Humanidad de si misma y de su instinto de autodestrucción.

Otra obra cinematográfica que definitivamente hace pensar, es AI (Inteligencia Artificial) (Spielberg, 2001), dirigida por Steven Spielberg. A mediados del siglo XXI, la empresa estadounidense Cybertronics crea el prototipo de un niño-robot capaz de mostrar amor por sus poseedores humanos. Un hijo-robot, llamado David, que tiene incluso un osito de peluche robot, que vela por su seguridad y siempre le acompaña. David evoluciona en sus sentimientos e incluso le lleva a dilemas existenciales: ¿Por qué él no puede llegar a ser un niño de verdad? El célebre Pinocho de Walt Disney lo consiguió, gracias al Hada Azul, ¿por qué no él? La amplia barrera que actualmente nos diferencia de las máquinas, se empieza a estrechar en la mente de David. Pero la barrera continúa y él no es capaz de afrontarlo...Los sentimientos...aquello que nos hace humanos... ¿Llegará un día en que los robots puedan sentir?

Algo parecido ocurre en el Hombre Bicentenario, protagonizado por Robin Williams y basado en el libro de Asimov del mismo nombre (Asimov, 1976). Andrew entra en casa de la familia Martin para colaborar en las tareas domésticas. Pronto se dan cuenta de que su robot es capaz de interpretar las emociones e incluso corresponder a ellas, a pesar de no haber sido pre programado para ello. Andrew va evolucionado, con ayuda de su familia humana, a la que ve envejecer, incluso morir. Gracias a sus actualizaciones consigue que sus órganos y sus funciones se asemejen a los humanos. Se casa con Portia y pone todo su afán en que se reconozca públicamente su humanidad, a pesar de sus orígenes, en el Congreso Mundial. Antes de asistir, Andrew consigue contaminar su sistema para poder empezar a degradarse y, algún día, morir.

En el Congreso, se produce la siguiente conversación con la presidente del mismo (Barnathan y Columbus, 1999):

-Siempre he intentado dar sentido a las cosas. Debe de haber alguna razón para ser como soy. Como puede ver Señora Presidenta ahora ya no soy inmortal.

- ¿Lo ha dispuesto todo para morir?

-En cierto sentido sí. Estoy envejeciendo y mi cuerpo se está deteriorando y, al igual que el de ustedes, al final dejará de funcionar. Como robot, podría haber vivido para siempre. Pero hoy les digo a ustedes, que prefiero morir como hombre que vivir toda la eternidad como máquina.

- ¿Por qué lo desea?

-Para que se me reconozca. Solo por quien soy y por lo que soy. Ni más, ni menos. No busco la aclamación ni la aprobación. Sino la simple verdad de dicho reconocimiento. Este ha sido el objetivo elemental de mi existencia y debo conseguirlo tanto si quiero vivir, como morir con dignidad.

-Señor Martin. Lo que usted está solicitando es tremendamente complejo y controvertido, no será una decisión fácil. Le pido que sea usted paciente.

-Entonces, esperaré su decisión, Sra. Presidenta.

Será en su lecho de muerte cuando, finalmente el Congreso Mundial reconozca su tan ansiada humanidad. 
En el mundo de Her (Ellison y Jonze, 2013) los sentimientos entre las máquinas y los humanos están a la orden del día. Ambientada en un futuro cercano, la trama se centra en un hombre que se enamora de Samantha, un sistema operativo -con una dulce voz femenina- basado en el modelo de Inteligencia Artificial, diseñado para satisfacer todas las necesidades del usuario. Entre ellos surgirá una extraña y apasionada relación sentimental en la que el mundo virtual y real se mezclan continuamente.

El futuro de la ingeniería genética es la base de la famosa película de Ciencia Ficción Blade Runner (Deeley y Scott, 1982), ambientada en una época en la que se fabrican humanos artificiales, a los que se denomina "replicantes", principalmente para emplearlos en trabajos considerados peligrosos o para enviarlos como esclavos a las colonias exteriores de la Tierra. Estos se asemejan físicamente a los humanos, aunque tienen una mayor agilidad y fuerza física, pero carecen de la misma respuesta emocional y de empatía. Los replicantes fueron declarados ilegales en el planeta Tierra tras un sangriento motín en Marte, donde trabajaban como esclavos. Un cuerpo especial de la policía, Blade Runners, se encarga de identificar, rastrear y retirar de la circulación a los replicantes fugitivos que aún se encuentran en la Tierra.

Las anteriormente descritas son solo algunas muestras de las innumerables películas de ciencia ficción, muchas veces basadas en libros de este mismo género, que han impregnado la cultura del siglo XX y sigue haciéndolo en el XXI. Algunas de sus imaginarias predicciones son actualmente una realidad. $Y$ los últimos avances de la tecnología auguran un futuro próximo en el que no sería improbable que muchos otros llegaran a ser palpables.

\subsection{La línea entre la Ciencia Ficción y la realidad comienza a difuminarse}

En el renombrado Massachusetts Institute of Technology (MIT) de Estados Unidos lo tienen claro. La imaginación, basada en datos científicos, es la clave para evolucionar hacia el futuro. Esta gran incubadora de los grandes inventos actuales no duda en recomendar a sus estudiantes la lectura de libros de Ciencia Ficción para que se inspiren a la hora de crear prototipos. En uno de sus laboratorios, profesores de física han recreado los sables de los Jedi de Star Wars (Gent, 2017), induciendo a los fotones a unirse para formar moléculas. Lo hicieron simplemente para probar que la Ciencia podía conseguirlo, al menos en un laboratorio. Y no son los únicos.

Esta vez evocando al momento en que la nave Enterprise de Star Trek entra en velocidad warp, superando la velocidad de la luz, el físico Harold “Soony” White, del Centro Espacial Johnson de la NASA cree que es posible conseguirlo sin violar las leyes de la física (Chitwood, 2016). Esta teoría ya había sido investigada con anterioridad, sin embargo, había sido descartada por no considerarse plausible. Se trataba de construir una nave espacial ovalada con un gran anillo alrededor. Este anillo, elaborad con una materia 'exótica', debía de tener la capacidad de modificar el espacio-tiempo alrededor de la nave, creando una región de espacio-tiempo comprimido al frente otra de espacio-tiempo expandido hacia atrás, todo esto sin modificar el espacio-tiempo de la nave en sí. Esto permitiría a la nave espacial moverse teóricamente a 10 veces la velocidad de la luz, sin romper las leyes de la física, siempre y cuando pudiéramos generar $10^{\wedge} 45$ Julios de energía. Teniendo en cuenta que esta cifra es científicamente inalcanzable, el proyecto se había abandonado. Sin embargo White cree haber encontrado la solución. El nuevo método consistiría en sustituir la forma del anillo, por una forma toroidal, lo que reduciría la energía necesaria para impulsar la nave al equivalente de la masa del Voyager 1, lanzado en 1977. Además, afirma que se podría 
reducir incluso más la cantidad de energía si se hace oscilar la intensidad de la modificación del espacio-tiempo en torno a la nave. Estas afirmaciones hacen plausibles la teoría imaginaria y abre un interesante campo de investigación científica. Siguiendo con estos desarrollos científicos, en principio únicamente imaginarios, en la Universidad de Delaware (EE.UU.), el profesor e ingeniero químico Norm Wagner desarrolló una sustancia capaz de repeler los ataques con cuchillo y absorber las vibraciones. Algo parecido al material ficticio que recubre el escudo del Capitán América, el Vibranium. Y los estudiantes de ingeniería química de la Universidad de Stanford (EE.UU.) generaron una piel sintética elástica que conduce electricidad, puede sentir el tacto, como la piel humana, y se cura a sí misma. No se puede usar para curar instantáneamente las heridas, como un mutante, pero esta revolucionaria piel tendría aplicaciones en vendajes de alta tecnología y ayudaría a controlar la salud del paciente. También podría usarse en productos como relojes inteligentes, donde la electrónica tiene que ajustarse a una superficie curva (Fussell, 2013). También el profesor estadounidense, James Kakalios, autor del libro La física de los superhéroes (Kakalios, 2006), apoya esta teoría y recuerda que la investigación científica y la Ciencia Ficción comienzan con las mismas palabras: 'Qué pasaría sí...' , por lo que tienen principios comunes que deben de tenerse en cuenta. En su obra plantea la siguiente hipótesis ¿Qué pasaría si los superhéroes salieran de las páginas de los cómics y se hicieran reales? ¿Podrían realmente trabajar sus maravillas en un mundo limitado por las leyes de la física? ¿Cuánta fuerza debería de tener Spiderman para saltar de un edificio a otro? ¿Podría Storm de los X-Men controlar el clima? Kakalios explora en su libro, a través de estos personajes, multitud de conceptos físicos, desde la energía hasta la termodinámica o la mecánica cuántica. Además, prueba cómo los comics a menudo han estado por delante de la ciencia al explicar temas relativamente recientes en mecánica cuántica (con Kitty Pryde de los X-Men) y la teoría de cuerdas (con la Crisis sobre las Tierras Infinitas). Esto también ocurre con los descubrimientos actuales relativos al control mental y la telequinesia que vimos en Carrie, en varios X-Men, o incluso en Iron Man. "Cuando Iron Man usa sus rayos X o sus botas de propulsión, no usa ningún comando de voz, ni pulsa ningún botón. Simplemente piensa en ello", explica Kakalios. Esta meta, el poder de incentivar nuestra mente hasta límites únicamente hasta ahora sospechados por la Ciencia Ficción, es uno de los grandes retos que persiguen los científicos en la actualidad. Cada vez se ven más avances en este campo. Unas innovaciones extremadamente útiles en el área de la Medicina ya que, entre otras cosas, podría ayudar a las personas con extremidades protésicas a controlarlas de forma más precisa e intuitiva.

Como vemos, soñar con mundos imaginarios del futuro, no tiene por qué ser solo sueños imposibles de realizar. En nuestras manos está arrastrarlos hasta nuestra realidad. La gran pregunta es, ¿iHasta dónde querremos llegar?

\subsection{Los primeros robots}

Los robots no son una tecnología del presente, ni del futuro, sino una evolución lógica de ideas pasadas generadas hace siglos. Desde la antigüedad, el Hombre ha sentido fascinación por las máquinas capaces de imitar movimientos o reacciones humanas. Ya en la antigua Grecia la mitología aducía una criatura gigante, llamada Talos, que Zeus regaló a la diosa Europa, en señal de su amor. Según este mito, la sangre de Talos era plomo, el mismo fluido divino que, según la mitología corría por las venas de los dioses. La única vena de este inmenso hombre de bronce iba desde el cuello, a través de su cuerpo, hasta uno de sus talones y allí la cerraba un clavo de bronce. Este era su único punto débil. Según la leyenda, Talos protegía a Creta de los posibles invasores. Para ello, cada día daba tres vueltas al perímetro de la isla, impidiendo entrar en ella a los 
extranjeros y salir a sus habitantes que no tenían el permiso del rey. Si alguno era sorprendido, se metía en el fuego hasta calentarse al rojo vivo y abrazaba a su víctima hasta calcinarla. Son muchos los teóricos que ven a Talos como una visión de los futuros soldados robot (Mazlish, 1995).

Desde entonces, multitud de científicos, a lo largo de la Historia, han colaborado en este proceso de evolución de la tecnología robótica, muchos de ellos incluso desde la inconsciencia. Arquímedes (287-212 a.C) no inventó robots, pero sí sistemas mecánicos que se utilizan hoy en la robótica, como la leva, la polea, el resorte o el tornillo. Y, en el siglo I a. C, Herón de Alejandría describió en su Tratado Automata (traducido del griego "Aquel que se mueve solo") una colección de dispositivos artificiales capaces de moverse de forma autónoma. Entre ellos, destacaban los que imitaban a aves que gorjeaban, volaban y bebían. El movimiento se articulaba gracias a la fuerza del agua, la gravedad o un sistema de palancas (Sáez Vacas, 1981).

Dando un salto en el tiempo y trasladándonos a la Era Medieval, en esta época, las figuras humanas con mecanismos ocultos estaban a la orden del día. Muchos eran usados en las iglesias para impresionar a los fieles campesinos y aumentar su fe en un poder superior. Por ejemplo, era habitual ver figuras de hombres mecánicos que podían dar la hora por si solos golpeando con su hacha sobre una campana.

El ingeniero árabe Al-Jazari (1136-1206) describió e ilustró en su 'libro del conocimiento de los ingeniosos mecanismos', dispositivos mecánicos, entre los que se encontraba un gran reloj de agua accionado por contrapesos y un autómata con forma de humanoide capaz de servir bebidas.

Incluso, el gran inventor y pintor Leonardo da Vinci diseñó, alrededor de 1945 un autómata humanoide que emula a un guerrero vestido con armadura medieval, capaz de mover brazos, cuello, mandíbula.., de una forma anatómicamente perfecta. Tal y como describe Antoni Escrig en su libro, El Reloj Milagroso (Escrig, 2014):

\begin{abstract}
"De proporciones anatómicas que seguían el establecido canon vitruviano, el androide tenía la apariencia externa de una armadura. Un conjunto de poleas, cables y engranajes accionaban los miembros del caballero mecánico permitiéndole andar, sentarse, mover las manos, mover la cabeza y la mandíbula. Disponía de dos sistemas de control independientes. El que se encargaba de accionar las piernas, permitiendo mover de forma independiente las caderas, tobillos y rodillas, era externo; un operario transmitía el movimiento mediante cables. En cambio, el control de los miembros superiores, que incluían hombros, codos, muñecas y manos, era programable mediante un árbol de leva”'.
\end{abstract}

Ya, en la primera mitad del siglo XVI, en España, el ingeniero Juanelo Torriano, Relojero de la Corte de Carlos I, construyó un autómata llamado "El Hombre de Palo", capaz de cruzar una de las calles de Toledo y pedir limosna. Hoy se ha convertido en leyenda, pero su existencia histórica está probada. Poco más de un siglo después, el ingeniero francés Jacques de Vaucanson ideó una serie de autómatas que actuaban como flautistas y tamborileros. Pero su más famoso mecanismo fue el llamado "Pato con Aparato Digestivo", un dispositivo dotado con más de 400 piezas móviles capaz de batir las alas, comer, realizar la digestión y defecar, tal como lo hace un pato vivo. Era el siglo XVIII y primeros autómatas complejos comenzaban a ser una realidad (Sanchez-Martin et al, 2007).

Proseguimos con nuestro camino por la Historia, esta vez dando pasos de gigante durante las Revoluciones Industriales. Ambas supusieron no solo un gran avance en ingeniería, ciencia y matemáticas, si no en la evolución de la sociedad en 
general. Muchas de las arduas tareas manuales comenzaron a ser reemplazadas por unas máquinas y motores a vapor cada vez más complejas y eficientes. Estas eran capaces de hacer en unos minutos y sin esfuerzo, lo que a varias personas podía llevarle incluso días. Por tanto, las fábricas comenzaron a emplear máquinas para aumentar la carga de trabajo o la precisión en la producción de muchos productos. Era el primer enfrentamiento entre el Hombre y las máquinas. El proceso fue largo. Una evolución y concienciación social pausada, en gran parte de los casos, que dio lugar a una adaptación aceptable por parte de los trabajadores. La mayoría se adaptaron a los nuevos puestos de trabajo que surgieron, aprovecharon sus ventajas y prosiguieron su camino de la mano de la evolución. Aquella vida era más fácil, les permitía entrar en una zona confort, muy diferente a las duras condiciones de su etapa anterior, por ejemplo, en las labores del campo (Terrén, 2000).

Entre los personajes más destacados de esta época que contribuyeron al impulso hacia la robótica actual cabe mencionar a Charles Babbage (1791-1871), quien trabajó para desarrollar los fundamentos de la informática en el siglo XIX. Uno de sus proyectos más exitosos fue el motor de diferencia y el motor analítico. Aunque nunca se completó debido a la falta de fondos, estas dos máquinas establecieron las bases para los cálculos mecánicos.

Asímismo, la pionera Ada Lovelace (1815-1852) desarrolló de una serie de instrucciones que permitían realizar cálculos en una versión rudimentaria de lo que hoy conocemos como ordenador. Era hija del gran poeta del romanticismo inglés Lord Byron, y fiel seguidora de Charles Babbage, con quien trabajó desde los 18 años. La máquina analítica en la que ambos trabajaron se pensó para que pudiera ejecutar diferentes algoritmos y resolver cualquier clase de problema. Durante este periodo de trabajo conjunto, Ada Lovelace concibió la idea de que la máquina ideada por su tutor pudiese ser reconfigurada para calcular números de Bernoulli a través del uso de tarjetas perforadas. En la actualidad, este proyecto, que se le atribuye, está considerado como el primer programa de ordenador, por lo que a Ada Lovelace se la reconoce como la primera persona en describir un lenguaje de programación, incluso cien años antes de que se fabricase el primer ordenador (Pazos, 2010).

Estos son únicamente algunos de los inventos más destacados relacionados con la tecnología robótica, anteriores al siglo XX. Así demostramos un proceso de evolución con unos sólidos cimientos forjados muchos siglos atrás. El siglo XX supuso una revolución tecnológica exponencial basada, sin duda, en los cimientos asentados siglos atrás. La creciente imaginación entorno a lo que aquellas máquinas que resolvían parte de nuestro trabajo podían llegar a hacer, comenzaron a mezclarse, de forma continuada, con las posibilidades reales de la evolución científica. La literatura alimentaba a la ciencia... Apareció el término "robot" de la mano del dramaturgo checo Karl Capek e Isaac Asimov acuñó y dio a conocer mundialmente el concepto de la "robótica" a través de sus famosas "3 leyes", a las que ya hemos aludido anteriormente.

La evolución del pensamiento científico en este campo, durante esta época, fue abrumador (Echevarría, 2015). El rápido desarrollo de grandes inventos tecnológicos permitieron comenzar a pensar en la posibilidad real de dotar a las "máquinas", a los "autómatas", de algo hasta entonces intrínseco únicamente en el ser humano: la inteligencia, la capacidad para pensar y razonar siguiendo una lógica. Alan Turing, considerado por muchos el padre de la Inteligencia Artificial, aludía a este concepto en su histórico artículo, publicado en 1950, en el que planteaba la pregunta: ¿Pueden las máquinas pensar? Para concretarlo propuso su "Juego de imitación", posteriormente conocido como "El test de Turing". Para este juego era necesario un interrogador (persona), ubicado en una habitación aislada, y en otra estancia una persona y una computa- 
dora. Ambos deberían responder a las preguntas que les realizase el interrogador de forma aleatoria. La máquina tenía que ser capaz de hacerse pasar por un ser humano; si el interrogador no podía distinguir entre el individuo y el ordenador, se consideraba que la máquina había alcanzado un determinado nivel de inteligencia. Para Turing, la inteligencia artificial comenzaba a existir cuando los humanos éramos incapaces de distinguir, en una conversación a ciegas, si nuestro interlocutor era también humano o máquina (Longo, 2010).

Muchos han sido los que, desde entonces, han intentado que sus programas informáticos superaran el 'Test de Turing'. En 1990 se creó el concurso Premio Loebner en el que intentaban superarlo, lo cual no fue posible hasta 2010 cuando el robot Suzette, de Bruce Wilcox, lo consiguió. Suzette, tenía 16.000 reglas de conversación y era capaz de mantener 40 horas de conversación ininterrumpida. Tenía una personalidad coherente y respondía emocionalmente. Hoy día, las teorías de Turing siguen aplicándose a los fundamentos de la robótica y la inteligencia artificial.

El estadounidense Marvin Minsky también revolucionó este campo durante el siglo XX y sus descubrimientos y pensamientos supusieron un antes y un después. En 1956 acuñó el acuñó el término "Inteligencia Artificial" -ya ideado por Turing- y estableció sus fundamentos guiado por el sueño visionario de dotar a los ordenadores con la capacidad para razonar. Él la definía de esta manera: "Es la ciencia de hacer que las máquinas hagan cosas que requerirían inteligencia si las hubiera hecho un humano” (Minsky, 2010). Dos años más tarde ingresó como profesor en el Massachusetts Institute of Technology (MIT), en Boston, donde trabajó más de cuatro décadas y donde creó su Laboratorio de Inteligencia Artificial, incubadora de muchos inventos robóticos actuales. Creó el primer prototipo de una máquina capaz de aprender de manera autónoma 'Snarc', diseñó las primeras manos con sensores táctiles y formó parte del equipo que diseñó ARPAnet, el precursor de lo que hoy conocemos como Internet.

Minsky dedicó gran parte de su tiempo a estudiar el cerebro y su comportamiento. Opinaba que transmitir esas características a una computadora "era solo cuestión de tiempo". En 1987 escribió el libro La Sociedad de la Mente, en el que explica el funcionamiento de la mente humana como pequeñas unidades o agentes que interaccionan hasta desarrollar tareas específicas. Según el autor, estos agentes carecen de 'inteligencia' propia, y no es hasta que interaccionan y se comunican cuando conforman la mente, con un aprendizaje evolutivo. Fue uno de los máximos defensores del transhumanismo, un movimiento filosófico e intelectual que considera que es necesario utilizar la tecnología para potenciar nuestras capacidades, evitar el sufrimiento y la vejez y, en última instancia, alcanzar la inmortalidad.

"Algún día seremos capaces de alcanzar la inmortalidad. Haremos copias de nuestros cerebros. Puede que los creemos en un laboratorio o que, simplemente, descarguemos su contenido en un ordenador" (Minsky, 1986).

La influencia de Minsky también se vio reflejada también en la Ciencia Ficción. Stanley Kubrick, uno de los grandes directores de cine del siglo XX, lo visitó en 1968 mientras preparaba 2001: Una odisea en el espacio, para que lo asesorara en el diseño del asistente virtual que traicionaría a la tripulación de su nave, HAL 9000. También fue asesor de películas como Parque Jurásico, Terminator, Inteligencia artificial y El hombre bicentenario.

La industria acogió estos avances en robótica de manera paulatina. En 1961, General Motors introdujo el primer robot industrial 'Unimate' para ayudar en la producción de automóviles. Fue desarrollado por Joseph Frederick Engelberger, considerado padre de la robótica industrial y fundador de la empresa Unimation, junto con su socio, el inventor George 
Devol. Unimation trabajó junto a General Motors para ampliar el uso universal de los robots en la industria y evitar que los operarios realizaran trabajos potencialmente peligrosos. La máquina de Engelberger se hizo muy popular a nivel internacional, y fue incluso protagonista de uno de los legendarios shows televisivos de Johnny Carson, en el que demostró que su robot era capaz de servir cerveza, dirigir una orquesta y tocar el acordeón. En definitiva, el Unimate supuso un antes y un después en cuanto a la implantación de la tecnología robótica en la industria e hizo una valiosa contribución a los avances tecnológicos del siglo XX. Desde entonces, los robots han sido utilizados en una variedad de aplicaciones susceptibles de ser duras o peligrosas para los humanos, incluyendo exploración del Espacio, uso militar e incluso para misiones de búsqueda y rescate (Valencia, 2004). Durante las siguientes dos décadas, los japoneses tomaron la iniciativa con una fuerte inversión en robótica. Mientras, en Estados Unidos y, sobre todo en Europa se miraba con preocupación cómo las fuertes inversiones en ambiciosos proyectos relacionados con estas tecnologías tardaban en producir frutos palpables y, sobre todo, rentables. En 1973, después de un controvertido debate en el Congreso de Estados Unidos sobre el tema, el matemático británico James Lighthill entregó un informe muy negativo sobre el estado de la Inteligencia Artificial y sus posibilidades en un futuro próximo, señalando que los descubrimientos hechos hasta entonces no habían cumplido, en absoluto, las expectativas prometidas. Muchos fondos para la investigación fueron suprimidos y, de esta manera, comenzó lo que se conoció como 'el invierno de la Inteligencia Artificial' (Lighhill, 1973).

Durante la década de los 90 comenzaron a retomarse algunos de los proyectos abandonados, especialmente en el MIT y en Stanford (EE. UU.), y en empresas privadas como Microsoft e IBM. Los ordenadores personales se habían popularizado y los "visionarios" en tecnología robótica e Inteligencia Artificial comenzaban a multiplicarse. En 1997 se produjo uno de los hitos en este campo. La supercomputadora creada por IBM 'Deep Blue’ se enfrentó al campeón mundial de ajedrez, Gary Kasparov, y ganó. Era la primera vez que una máquina vencía a un ser humano en una competición.

Desde entonces, la competencia entre el hombre y la máquina no ha cesado. De hecho crece actualmente a un ritmo exponencial, tanto esto, como las cuestiones éticas derivadas de esta comparación.

\section{Estado de la cuestión}

La IA es una realidad palpable en la actualidad. La recuperación económica a nivel mundial, en los últimos años ha propiciado el aumento de la inversión en este campo y, gracias a esto, a su desarrollo exponencial.

Actualmente estamos en los inicios de la que ya llaman la Cuarta Revolución Industrial (Granell, 2016). Un nuevo y trascendente paso en la Historia. El mundo interconectado que nos proporciona Internet, la Inteligencia Artificial y la Robótica modificará en breve la rutina de nuestras vidas, si es que no lo está haciendo ya. La investigación en estos campos avanza a gran velocidad, deslumbrándonos con descubrimientos que, hasta ahora, formaban parte de la Ciencia Ficción. La comunidad científica está entregada en su cometido y sueña con un mundo futuro cada vez más palpable. Sin embargo la Sociedad, el campo real de experimentación de esta Revolución, aquellos que finalmente tendrán que convivir con los robots y la IA ¿está preparada para un cambio tan drástico?

Estudios recientes (Hawksworth, J. et al, 2017) señalan que aquellos sectores cuyas tareas sean más mecánicas serán los más afectados. Fácilmente cualquier IA, o incluso un robot sin IA, puede suplantar este trabajo, reducir costes, e incremen- 
tar exponencialmente la productividad. Un robot no para para comer, un robot no duerme, un robot no coge vacaciones... un robot no protesta si no se le paga convenientemente. Cada vez son más las empresas que apuestan por esta inversión. Y es que a las IA pueden manejar el llamado Big Data y extraer conclusiones con extrema rapidez y precisión en los resultados. Lo que coinciden todos los expertos en señalar es que aquellos sectores en los que se vea implicado un componente emocional van a ser los que más tarde se vean alterados por las AI (Sirvent, 2017). Ese componente de cercanía, de psicología humana que tanto valoramos en muchas ocasiones, por ahora, no es previsible que puedan hacerlo las máquinas (García, 2017). Respecto a las regiones hay países más propensos a la implantación exitosa de las nuevas tecnologías (Hawksworth, J. et al., 2017). Por ejemplo, Japón. Allí ya conviven con AI y Robots, y prosiguen con grandes perspectivas de negocio las investigaciones al respecto. En EE.UU. también se espera una mayor implementación que en España por la aceptación social que históricamente suele tener la tecnología en este país. Allí ya están probando coches autónomos y el potencial comprador es abrumador. El periodismo está siendo uno de los sectores más claramente afectados por esta tecnología, dado el hecho de que genera una mayor productividad y eficiencia en la producción de contenidos (Papadiminiou, 2016). Actualmente son muchas las noticias generadas por máquinas, principalmente en medios de comunicación norteamericanos como The Washington Post, The Wall Street Journal, Forbes y CNN. Según el último informe del Instituto Reuters Journalism, Media, and Technology Trends and Predictions (Newman, 2018) una vez más el mundo del periodismo está destinado a acoger la tecnología emergente, tal y como sucedió en su momento con la radio, la televisión, e Internet. Aunque aún nos encontramos en los albores de este nuevo tiempo de periodismo dominado por la Inteligencia Artificial, el fin está claro. Se tiende a la automatización de tareas y al llamado 'Periodismo aumentado", tal y como define en su último informe Associated Press, titulado: Cómo la Inteligencia Artificial impactará al periodismo: una guía para redacciones en la Era de las máquinas inteligentes". (Marconi, 2017). Actualmente ya se están vislumbrando las ventajas de la implantación de las IA. El periodista delega labores de documentación y análisis a la máquina, la cual es capaz de resolver de forma precisa y rápida. Incluso es capaz de escribir noticias sencillas a partir de datos de fuentes de información pre fijadas por el programador. Sin embargo, se están encontrando claros inconvenientes. Uno de los principales deriva del campo de la ética. La muchas veces cuestionada ética del periodista cobra una nueva dimensión cuando es supuestamente una 'máquina' la que toma las decisiones. Este es uno de los grandes campos de investigación en la actualidad. El objetivo: intentar que las IA se integren en el periodismo sin desvirtuar la profesión, principalmente desde un punto de vista ético.

\section{Objetivos}

El objetivo general de la investigación para este artículo es analizar el impacto que las nuevas tecnologías y, en particular, la Inteligencia Artificial y la robótica tienen en el campo del periodismo. Asimismo, intenta pronosticar, basándose en la evolución histórica del concepto estudiado y en la influencia de la Ciencia Ficción sobre la conciencia social, cuál será el futuro del periodismo y de sus profesionales una vez que acoja definitivamente las tecnologías relacionadas con la Inteligencia Artificial. 


\subsection{Objetivos específicos:}

1. Analizar la evolución histórica de los robots y de la Inteligencia Artificial, con el objetivo de comprender la evolución del pensamiento sobre esta tecnología.

2. Conocer las principales obras, tanto literarias como cinematográficas, que han contribuido a crear la idea actual que la sociedad tiene de los robots y de la IA.

3. Identificar el punto de conexión entre realidad y ficción en referencia a las nuevas tecnologías de IA y robótica.

4. Conocer los intentos actuales de implementación de tecnologías de IA en el campo del periodismo.

5. Identificar las ventajas e inconvenientes de la implantación de esta tecnología. Conocer el rol de los periodistas en esta nueva etapa en la que deberá convivir profesionalmente con 'la máquina'.

6. Desvelar las perspectivas de futuro de la IA en el campo del periodismo.

Se pretende, con este estudio, identificar las principales tendencias de la investigación y aspectos relevantes conocidos, sirviendo de referencia y orientando la reflexión entre la comunidad científica acerca de las nuevas oportunidades que las tecnologías IA están trayendo en la actualidad, y traerán en mayor proporción en un futuro próximo, al campo del periodismo. Se pretende dar a conocer la situación actual, así como sugerir aspectos o temas de investigación futuros que aún están por definir.

\section{Metodología}

En línea con los objetivos planteados, se ha realizado una revisión de artículos científicos, libros e informes que abordan algunas de las diferentes dimensiones del impacto social de la tecnología en general, y de la Inteligencia Artificial y la robótica en particular. La identificación y recopilación de documentos de ámbito académico se ha realizado siguiendo la metodología de revisión Sistematizada, que se caracteriza por cuatro dimensiones muy bien determinadas por la filosofía de la que procede (las systematic reviews), que aportan rigor a la consulta y que se especifican en la siguiente tabla (Codina, 2017):

\begin{tabular}{|l|l|l|l|}
\hline SISTEMÁTICA & COMPLETA & EXPLÍCITA & REPRODUCIBLE \\
\hline $\begin{array}{l}\text { No es arbitraria: ni sesgada } \\
\text { ni subjetiva, sino que, por el } \\
\text { contrario, se examina la mejor } \\
\text { producción científica dispo- } \\
\text { nible utilizando las mejores } \\
\text { fuentes de información. }\end{array}$ & $\begin{array}{l}\text { Se han usado sistemas de } \\
\text { información de los que se } \\
\text { presume que facilitan el acce- } \\
\text { so virtual a la totalidad de la } \\
\text { producción de calidad de una } \\
\text { disciplina. }\end{array}$ & $\begin{array}{l}\text { Implica que se dan a conocer } \\
\text { tanto las fuentes utilizadas } \\
\text { como los criterios de búsque- } \\
\text { exclusión de los artículos. }\end{array}$ & $\begin{array}{l}\text { Permite a otros investigadores } \\
\text { comprobar el trabajo y, si lo } \\
\text { desean, seguir los pasos y } \\
\text { contrastar los resultados para } \\
\text { determinar su exactitud o su } \\
\text { grado de acierto }\end{array}$ \\
\hline
\end{tabular}

Fuente: elaboración propia

Para la investigación bibliográfica sistematizada se ha accedido a la base de datos bibliográfica Scopus, la cual avala la calidad de estas publicaciones (De Granda-Orive et al., 2013). Se trata de la mayor base de datos de resúmenes y de literatura revisada por pares y cuenta con herramientas inteligentes que permiten controlar, analizar y visualizar investigación académica. Dada su amplia cobertura, tanto geográfica como temática, se la considera ideal para ser utilizada para revisiones 
bibliográficas, incluyendo las de tipo sistematizadas en cualquier área, pero de forma muy especial en Ciencias Humanas y Sociales (Codina, 2018). Los criterios de búsqueda principal han sido delimitados por palabras clave incluyendo operadores booleanos de inclusión (AND) o exclusión (NOT). También se han usado filtros de rangos de fechas. Asimismo, se han aplicado estos mismos criterios de búsqueda en la Web of Science, donde se detallan las referencias de las principales publicaciones científicas de cualquier disciplina del conocimiento, tanto científico como tecnológico, humanístico y sociológicos desde 1945. También ha sido consultado Dialnet, portal de difusión producción científica hispana, creado por la Universidad de La Rioja (España), especializado en ciencias humanas y sociales. Consideramos su base de datos de gran valía, ya que no solo incluye los índices de las revistas científicas y humanísticas de España, Portugal y Latinoamérica, así como libros (monografías), tesis doctorales, homenajes y otro tipo de documentos, sino que, además, da acceso, de forma gratuita, al texto íntegro de muchos de ellos (Magriñá Contreras, 2007).

Además, ha sido de gran ayuda para la adquisición de los conocimientos necesarios para esta investigación, la colaboración de la autora en el foro online Internacional Ethically Aligned Design (EAD), en el que profesionales de relevancia internacional exponen su punto de vista sobre cómo crear el mejor código ético de la Inteligencia Artificial. Así como en los foros de discusión de la European AI Alliance, organismo dependiente de la Comisión Europea, de la que la autora también es miembro.

Esta investigación se ha sustentado, además, en las conclusiones de la participación de la autora, como ponente activo, en el IX Seminario Internacional de Lengua y Periodismo celebrado en San Millán de la Cogolla, titulado “El español del futuro en el periodismo de hoy", con una ponencia sobre el papel de las máquinas en el proceso periodístico. En este evento la autora pudo definir las hipótesis planteadas en este trabajo ayudada por las fundamentadas opiniones de expertos en la materia, tanto del mundo académico, como empresarial.

Además, la visión de conjunto de la investigación se ha completado gracias a la asistencia presencial de la autora a numerosos congresos, seminarios y conferencias relacionados con el impacto de la IA en los puestos de trabajo y en la sociedad en general, entre ellos.

Los conocimientos de la autora necesarios para la realización de este artículo derivan, además, de las diversas investigaciones relacionadas con otros ámbitos del impacto social de la Inteligencia Artificial, adquiridos como investigadora principal del grupo SIMPAIR (Social Impact of Artificial Intelligence and Robotics), así como de una experiencia profesional de 17 años trabajando en los medios digitales del grupo PRISA, durante los cuales la autora pudo observar, comprender y analizar, de primera mano, la gran influencia de la tecnología en el periodismo en general, y en los periodistas, en particular.

\section{Análisis y resultados de la investigación}

La historia de la evolución de la robótica y la Inteligencia Artificial, influida significativamente en los últimos tiempos por la literatura y el mundo cinematográfico, ha propiciado, sin duda, el momento que estamos viviendo en la actualidad, como se puede apreciar en los primeros apartados de este artículo. 
La ciencia ficción que muestra escenarios de IA ha creado prejuicios sociales hoy muy visibles a todos los niveles cuando se habla de estas tecnologías. Tendemos a ofrecer a estos algoritmos características humanas, incluso conciencia sobre el bien y el mal. Asimismo, en nuestra imaginación la línea con la realidad, en este aspecto, está muy difuminada. Son muchos gurús los que auguran la revolución de los robots, la llamada Singularidad tecnológica, en la que estos últimos superarán en inteligencia a los humanos y se convertirán en la "raza dominante" en la Tierra", así como potenciales usos 'maliciosos' de esta tecnología que pudieran propiciar desastres considerables. (Brudage et al, 2018).

Aunque esta Singularidad Tecnológica aún parezca una utopía inalcanzable, dado el rápido avance de las tecnologías en la actualidad no podemos emplazar la cuestión como algo inalcanzable. Como se ha demostrado a lo largo de la Historia, cualquier invento que hoy parece ciencia ficción mañana puede ser una realidad tangible.

Según explica la ciborgantropóloga del Massachussets Institute of Technology (MIT), Amber Case, uno de los principales problemas a los que nos enfrentamos en la actualidad es que la tecnología avanza con demasiada rapidez, y nos cuesta asumir tanto cambio. "En apenas unos años, un ordenador ha pasado de ocupar una habitación a caber en nuestro bolsillo y conectarnos con todo el mundo” (Ventura, 2018). Es el llamado 'Shock tecnológico', en el que se mezcla el miedo a lo desconocido y una vaga conciencia de sus ventajas e inconvenientes.

En este sentido, también Robert Ornstein, psicólogo, comenta en su libro La evolución de la conciencia un hecho recalcado por numerosos investigadores actuales en estas tecnologías: "Desde la aparición de los seres humanos hasta mi nacimiento, en 1942, un largísimo período fue necesario para que la humanidad alcanzara una población de 2650 millones de seres humanos. Sin embargo, los pocos años de mi vida fueron suficientes para agregar a la humanidad viviente un número igual de personas... Si la producción de los primeros mil millones de seres humanos necesitó aproximadamente de un millón de años, para los siguientes mil millones fueron suficientes 14 años... y el ritmo se acelera" (Ornstein, 1991).

Por su parte Fernando Iglesias destaca también en su libro La modernidad global: Una revolución copernicana en los asuntos humano este ritmo acelerado en el que nos encontramos: "Desde que los fenómenos acelerados impactaron en los procesos sociales, la futurología se transformó en una ciencia de escasa certidumbre pero de importancia creciente. Con el diario del día siguiente en la mano resulta fácil burlarse delas profecías erradas de sus gurúes. Menos simple es comprender la razón: en un mundo en cambio lento, el futuro cercano era previsible y el futuro lejano, imprevisible. Pero en un mundo sometido al cambio acelerado, el futuro cercano es imprevisible, y el lejano incomprensible, tan incomprensible como si tuviéramos que explicarles a nuestros tatarabuelos el concepto de computación en la nube" (Iglesias, 2011).

Este cambio acelerado también ha afectado significativamente al mundo del periodismo. No a los principios básicos del periodismo que, que continúan siendo los mismos, sino más bien a la forma que tienen los periodistas de hacer periodismo. Y la corta transición no está siendo sencilla para estos profesionales.

\subsection{La rápida evolución del periodismo}

Las últimas décadas del siglo XX y, por ahora el siglo XXI, ha supuesto una gran revolución a nivel periodístico. Internet es para el periodista una fuente de información y documentación infinita (Salazar García, 2003). Desde el nacimiento de la World Wide Web, en 1991 -que facilitó el acceso de todo tipo de usuarios a Internet-, el crecimiento de datos desestructu- 
rados en la Red de redes ha sido exponencial. Anteriormente a su aparición, eran los periodistas los que proporcionaban la información que consideraba relevante para los lectores. Ahora estos últimos pueden extraer de la Red lo que les parece más interesante, a través de webs temáticas, periódicos digitales, blogs y foros, entre otros. Las posibilidades son muchas y no siempre fiables, teniendo en cuenta el hecho de que cualquiera, con conexión a Internet, puede publicar cualquier cosa, sin necesariamente ser veraz.

Podría decirse que el trabajo del periodista, como tal, ha dejado de ser necesario. Sin embargo, es justamente en este último punto en el que está su nueva labor: Estructurar el 'caos' de datos, aportar fiabilidad a las noticias que cuenta sustentándose en la marca y prestigio de su medio, proporcionar enlaces a otras webs relevantes para la noticia, aportar recursos multimedia disponibles en Internet que la complementen... y no dejarla morir ahí, sino también aportar recursos que se actualicen automáticamente en tiempo real: tipo noticias de la Bolsa, el Tiempo... También dar opción a sus lectores de aportar sus opiniones al respecto marcando su propia línea editorial.

Todo esto es la labor del periodista digital en la actualidad, pero ¿̇hacia qué tiende esta profesión? El tremendo potencial que tiene Internet para los periodistas tan sólo se ha atisbado en España. En 1999, época del boom de los portales de Internet todos confiaban y soñaban con las grandes posibilidades que ofrecía e intentaron sacar la mayor rentabilidad posible. Pero el nuevo soporte requería algo más importante: un cambio de mentalidad, no sólo social, sino también empresarial. Introducir nuevas tecnologías implicaban fuertes inversiones en investigación, confianza en los proyectos a medio-largo plazo, menos burocracia que suponga ralentizar algo que requiere continuidad y agilidad mental de los implicados. Algo que, como hemos explicado anteriormente, es complicado debido a la rápida evolución de la tecnología. La crisis del periodismo actual es, en gran parte, fruto de esta falta de adaptación, motivado por un cambio demasiado rápido, dentro de una sola generación. (Martinez Sánchez, 2012).

A pesar de estos inconvenientes, la evolución de la tecnología ha seguido marcando a la profesión y actualmente el gran reto es impregnar a las redacciones de 'inteligencia artificial' (Daewon y Seongcheol, 2018)

Según el último informe de Associated Press How Artificial Intelligence will impact journalism (Marconi, 2017), cada vez son más los medios de comunicación que están apostando por la IA para salir de la crisis, incorporando esta tecnología a sus distintos departamentos -desde el marketing, a la redacción y al diseño- logrando automatizar muchas de sus tareas con gran eficacia.

De hecho, el diario estadounidense The New York Times ha incorporado técnicas de aprendizaje automático o machine learning para buscar patrones en los datos de financiación de sus campañas publicitarias, con el objetivo de optimizar sus resultados. Además, emplean una IA para producir y gestionar contenidos. Asimismo, Los Angeles Times tiene su IA llamada "Bot Quake" que se encarga, sin intervención humana, de publicar noticias online en el momento en el que se detecta un terremoto en la ciudad y sus alrededores.

Por su parte, Associated Press lleva varios años utilizando Automated Insights (herramienta IA que permite el uso del lenguaje natural) para generar presentaciones de cualquier tipo: desde informes de ganancias de empresas públicas hasta clasificaciones de las ligas de béisbol. Su último informe constata que la automatización de estos trabajos ha permitido 
a la plantilla del medio contar con un $20 \%$ más de tiempo, que está siendo invertido en la elaboración de reportajes más extensos y profundos (Marconi, 2017).

Asimismo, en este mismo estudio, Marconi afirma que la IA transformará por completo la sala de redacción y el trabajo de los periodistas, creando una demanda de nuevas habilidades técnicas entre estos profesionales, y la necesidad de nuevos y mejores flujos de trabajo. "Los cambios no son drásticos, pero es importante tenerlos en cuenta al implementar AI en una sala de redacción”, dice. Los papeles de la redacción como “Editor de automatización” y “Periodista computacional” surgen junto con un mayor interés en cursos de periodismo digital y de datos, lo que significa que pronto ya no será suficiente para los periodistas realizar entrevistas o escribir artículos.

Marconi expone un cambio en el flujo de trabajo del periodista (Marconi, 2017)

\begin{tabular}{|c|c|}
\hline Flujo de trabajo tradicional & Flujo de trabajo con la incorporación de las IA \\
\hline $\begin{array}{ll}- & \text { Reportero } \\
\text { - } & \text { Editor de la noticia } \\
\text { - } & \text { Jefe de sección } \\
\text { - } & \text { Corrector }\end{array}$ & $\begin{array}{ll}\text { - } & \text { Reportero } \\
\text { - } & \text { Reportero +IA } \\
\text { - } & \text { Editor de la noticia + Editor IA } \\
\text { - } & \text { Jefe de sección } \\
\text { - } & \text { Corrector }\end{array}$ \\
\hline
\end{tabular}

Fuente: elaboración propia

Además, el informe de Associated Press sostiene que los cambios en la sala de redacción no solo tendrán un gran impacto en la forma en que los periodistas cubren una historia, sino también en la forma en que se presentan las noticias y se entregan a los lectores. Explica Marconi que hoy día el contenido del periódico único para todos: un periodista escribe una historia con la esperanza de llegar al mayor número posible de personas», dice. «El problema con este enfoque es que cualquier artículo dado solo generará interés de un subconjunto de lectores muy específico; aquellos que tienen afinidad con las ideas y el contexto presentado por el periodista". Marconi sostiene que esto está cambiando rápidamente, a medida que las compañías de medios se apresuran a identificar formas de aprovechar AI para generar muchas versiones de la misma historia. "Algún día", dice, "el contenido se adaptará a la personalidad individual, el tono, la ubicación, la hora del día y más, resolviendo finalmente la crisis en el compromiso del contenido". Del informe se desprende claramente la tendencia del periodismo en la actualidad, una vez incorporadas, las tecnologías IA: la personalización de la información, en función de la demanda de cada usuario.

\subsection{La ética y la privacidad de los datos, principales riesgos}

A pesar de las indudables ventajas que aportan estas nuevas tecnologías no solo al campo del periodismo, sino al resto de áreas, también existen riesgos importantes que están actualmente a debate derivados de la ética de los programadores de la IA y, en el futuro próximo, de la propia IA, y de la privacidad de los datos de los usuarios. Hay que tener en cuenta que los algoritmos tienen la capacidad de "aprender" de lo que hacen (machine learning) de lo que se van encontrando en el desarrollo de su trabajo, de su contexto, por lo que puede llegar un momento en que llegue cierta desvinculación respecto a su programación inicial. Por este motivo cierto control, ciertas normas y reglas, cierto seguimiento es necesario. En este 
sentido, cada vez son más las instituciones que abogan por un organismo internacional que regule el llamado Big Data y las actuaciones de las IA (Lopez de Mantarás, 2017).

En este sentido el Parlamento Europeo (PE), tras una propuesta de borrador (junio 2016) y un informe razonado (febrero 2017) ha aprobado un informe sobre Robótica en el que se establece un Código Ético de Conducta (European Parliament, 2017).

La propuesta de resolución del PE establece que es necesario establecer "un marco de guía ético para el diseño, producción y uso de los robots" que sirva de complemento a las diferentes recomendaciones puramente legales que se realizan. Es decir, profundizar en una nueva disciplina, la "roboética". La idea de fondo es que los estándares éticos deberían ir dirigidos a la humanidad -esto es, los diseñadores, productores y usuarios de los robots- y no tanto a los robots en sí mismos. Nathalie Nevejans, responsable del informe indica que no se debe confundir la ética en la robótica con la ética en las máquinas, es decir, una ética que obligue a los propios robots a adherirse a reglas éticas. Existen varios principios fundamentales que han sido recogidos por la resolución que incluyen la protección de la dignidad humana, la privacidad, la libertad, la igualdad de acceso o los efectos sociales, entre otros:

- Proteger a los humanos del daño causado por robots: la dignidad humana.

- Respetar el rechazo a ser cuidado por un robot.

- Proteger la libertad humana frente a los robots.

- Proteger la privacidad y el uso de datos: especialmente cuando avancen los coches autónomos, los drones, los asistentes personales o los robots de seguridad.

- Protección de la humanidad ante el riesgo de manipulación por parte de los robots: Especialmente en ciertos colectivos -ancianos, niños, dependientes- que puedan generar una empatía artificial.

- Evitar la disolución de los lazos sociales haciendo que los robots monopolicen, en un cierto sentido, las relaciones de determinados grupos.

- Igualdad de acceso al progreso en robótica: Al igual que la brecha digital, la brecha robótica puede ser esencial.

- Restricción del acceso a tecnologías de mejora regulando la idea del transhumanismo y la búsqueda de mejoras físicas y/o mentales.

Como se puede apreciar o a lo largo de este trabajo de investigación, la robótica y la IA afectarán en profundidad a todas las áreas del conocimiento y las relaciones sociales, y aún quedan muchas preguntas por contestar, entre ellas ¿Cómo será la IA en un futuro próximo? ¿Tendremos un Código Ético Internacional para la IA? Estas preguntas generan incertidumbre, pero el hecho está claro: la IA se va a seguir desarrollando a un ritmo exponencial y debemos pensar con rapidez la forma de gestionar este cambio.

\section{Conclusiones. Un momento de cambio para el periodista, no para el periodismo}

El periodismo es un área que se verá afectada significativamente por la evolución de las tecnologías relacionadas con la IA, las cuales cambiarán definitivamente la forma de enfrentarse a la profesión. La clave está en avanzar juntos. Los robots y los humanos. 
En este sentido el perfil del periodista cambia. Este profesional deberá formarse no solo en el conocimiento de cómo transmitir de forma adecuada la información, si no en la forma de colaborar adecuadamente con las máquinas IA para obtener el mejor resultado.

Es deber y labor del periodista, tanto en la actualidad como en un futuro inmediato, transmitir y 'traducir' con un lenguaje inteligible los avances tecnológicos en IA que tanto están conmocionando a ciertos sectores de la sociedad, que pudieran considerarse más débiles y menos predispuestos al cambio. Asimismo, deberá transmitir sus ventajas e inconvenientes de forma adecuada.

Estamos atravesando un momento de la Historia de avances tecnológicos exponenciales. El hecho de que estén ocurriendo sin que haya cambio generacional está motivando cierto rechazo, por falta de entendimiento de una parte de la sociedad más acostumbrada a la estabilidad.

La IA y la rápida evolución de tecnologías del futuro se espera que traigan cierta inestabilidad propiciada por esta rápida evolución. Esto conllevará a que tanto el profesional de la información, como el de cualquier otra área, tenga que estar aprendiendo y reciclándose durante toda su vida profesional. Es y será aún más a medida que avanzamos hacia el futuro, una condición necesaria del perfil de cualquier trabajador. Todo aquel que no lo acepte, se quedará fuera del sistema.

La sociedad debe de aceptar la IA como una tecnología más que ayudará a mejorar su estilo de vida. Como toda tecnología su uso inadecuado puede derivar en peligros, principalmente derivados del mal uso de los datos.

En este sentido, la IA conlleva unas consideraciones éticas que no tienen otras tecnologías. El hecho de dotar de 'inteligencia' a una máquina entra en conflicto directo con el concepto de hombre y las definiciones filosóficas y religiosas del término. Asimismo, la sociedad, como masa, tiende, por su idea preconcebida derivada de los mundos imaginarios de la Ciencia Ficción, tanto en novelas, como cine y televisión, a identificar la IA con connotaciones negativas. Es necesario un código ético internacional que regule convenientemente las implicaciones éticas que, tanto ahora, como en un futuro inmediato, vayan derivándose de las IA. En caso contrario, la intrusión de esta tecnología en la sociedad será muy escasa y de poco impacto, ya que generará rechazo.

\section{Referencias bibliográficas}

Asimov, Isaac (1942): Runaround. Nueva York: Street \& Smith.

Asimov, Isaac (1950): Yo, Robot. Barcelona: Edhasa.

Asimov, Isaac (1976): El hombre Bicentenario. Madrid: MR Ediciones.

Barnathan, M y Columbus, Chris (1999): El hombre bicentenario. Estados Unidos, Touchstone Pictures.

Berlanga de Jesús, A. (2016) "El camino desde la Inteligencia Artificial al Big Data”. Revista de Estadística y Sociedad. n. 68, pp. 9-11.

Brockman, John (1996): La tercera cultura. Más allá de la revolución científica. Barcelona: Tusquets.

Brudage, M. et al (2018): “The Malicious Use of Artificial Intelligence: Forecasting, Prevention. 
and Mitigation". Future of Humanity Institute. University of Oxford. [Disponible en: https://imgl.wsimg.com/blobby/go/ 3d82daa4-97fe-4096-9c6b-376b92c619de/downloads/1c6q2kc4v_50335.pdf] [Consultado el: 01/05/2018].

Campbell, Jeremy (1990): The improbable machine. What new discoveries in artificial intelligence reveal intelligence about how the mind really Works. Nueva York: Simon \& Schuster.

Chitwood, Adam (2016): NASA Has Designed a Warp Ship Inspired by 'Star Trek's Enterprise. Collider. [Disponible en: http://collider.com/star-trek-warp-ship-nasa-images/\#ixs-enterprise] [Consultado el: 14-04-2018].

Codina, Lluís (2017): Revisiones sistematizadas y cómo llevarlas a cabo con garantías: systematic reviewsy SALSAFramework. [Disponible en: https://www.lluiscodina.com/revision-sistematica-salsa-framework/] [Consultado el: 14-09-2018].

Codina, Lluís (2018): Scopus: caracterización y guía de uso avanzado Preparación, búsqueda y exportación de resultados. [Disponible en: https://www.lluiscodina.com/scopus-analisis-guia-utilizacion/] [Consultado el: 14-09-2018].

García, M (2014): “La Inteligencia Artificial en el cine: terriblemente humanos”. Crítica, n.993, pp.72-76.

Granell, F (2016): Los retos de la cuarta revolución industrial. Perspectivas económicas frente al cambio social, financiero y empresarial: solemne acto académico conjunto con la Universidad de La Rioja y la Fundación San Millán de la Cogolla / coord. por Real Academia de Ciencias Económicas y Financieras, pp: 57-74.

Daewon, k. y Seongcheol, k (2018) Newspaper journalists' attitudes towards robot journalism. Telematics and Informatics. Vol. 35. pp: 340-357.

Deeley, M y Scott, R (1982): Blade Runner. Estados Unidos:Blade Runner Partnership.

De Granda-Orive, J.I; Alonso-Arroyo, A.; García, Río; Solano-Reina, Jiménez-Ruiz, C.A.; Alexandre-Benavent, R (2013): Ciertas ventajas de Scopus sobre Web of Science en un análisis bibliométrico sobre tabaquismo, Revista Española de Documentación Científica, 36 (2), e011.

Diakopoulo, Nicholas (2014): Algorithmic Accountability. University of Maryland, USA [Disponible en: http://www.nickdiakopoulos.com/wp-content/uploads/2011/07/algorithmic_accountability_final.pdf] [Consultado el: 01-05-2018]

Echevarría, J (2015): “De la filosofía de la ciencia a la filosofía de las tecno-ciencias e innovaciones”. Revista iberoamericana de ciencia y tecnología. vol.10 no. 28.

Ellison, M y Jonze, S: Her. Estados Unidos: Annapurna Pictures.

Escrig, Antoni (2014): El Reloj Milagroso, y otras historias científicas sobre robótica , automática y máquinas prodigiosas. Barcelona: Almuzara.

European Parliament (2017): Report with recommendations to the Commission on Civil Law Rules on Robotics. [Disponible en:http://www.europarl.europa.eu/sides/getDoc.do?type=REPORT\&reference=A8-2017-0005\&language=EN] [Consultado el: 07/03/2018].

Fussell,J.(2013): Science fiction inspires real-world innovation. The Bulletin. [Recuperado de: https://www.bendbulletin. com/news/1284143-151/science-fiction-inspires-real-world-innovation] [Consultado el: 12/02/2018]. 
García, C.(2017). Principios para la Era Cognitiva. Jornadas Yo, Robot: puestos de trabajo que van a desaparecer. Madrid: ESIC.

Gent, E (2017): “Star Wars' Tech: 8 Sci-Fi Inventions and Their Real-Life Counterparts”. Live Science. [Recuperado de: https://www.livescience.com/58943-real-life-star-wars-technology.html].

González-Jiménez, H.(2018): "Taking the fiction out of science fiction: (Self-aware) robots and what they mean for society, retailers and marketers". Futures n.98, pp. 49-56.

Hawksworth, J.; Berriman, R and Goel, S. (2017):Will Robots really steal our jobs?: An international analysis of the potential long term impact of automation. Price Water house. [Disponible en:https://www.pwc.co.uk/economic-services/assets/international-impact-of-automation-feb-2018.pdf] [Consultado el: 17/01/2018].

Hurd, G.A and Cameron, J (1984): Terminator. Estados Unidos: Hemdale Film.

Iglesias, Fernando A (2010): La modernidad global: Una revolución copernicana en los asuntos humanos. Editorial Sudamericana.

Jaemin, J.; Haeyeop, S.; Hyunsuk, I. (2017): “Intrusion of software robots into journalism: The public's and journalists' perceptions of news written by algorithms and human journalists". Computers in Human Behavior. n. 71, pp. 291-298.

Jubak, Jim (1993): La máquina pensante. El cerebro humano y la inteligencia artificial. Barcelona: Ediciones B.

Kakalios, J. (2006): La física de los superhéroes. Madrid: Ma Non Troppo.

Kurtz, G y Lucas, G (1977): Star Wars. Estados Unidos: LucasFilm.

Lighthill, J. : Artificial Intelligence: a paper symposium. [Disponible en: http://www.chilton-computing.org.uk/inf/literature/reports/lighthill_report/contents.htm] [Consultado el: 02-04-2018]

Longo, G. (2010): El Test de Turing: "historia y significado": Novática: Revista de la Asociación de Técnicos de Informática, n.206, pp. 63-74.

López de Mántarás, R. (2017) Ética en la inteligencia artificial. Investigación y Ciencia. n. 491. pp. 49-49.

López Pellisa, T (2013): “Autómatas y robots: fantoches tecnológicos en R.U.R. de Karel Capek y El señor de Pigmalion de Jacinto Grau". Anales de la literatura española contemporánea, n.38, pp.137-159.

Magriñá Contreras, M (2007): La difusión de la producción científica española a través del proyecto DIALNET. Boletín de la ANABAD, Tomo 57, No 4, pp. 9-22.

Marconi, F., Siegman, A. and Machine Journalist (2017): The Future of Augmented Journalism: A guide for newsrooms in the age of smart machines. Associated Press. [Disponible en: https://insights.ap.org/uploads/images/ap_insights_the_future_of_augmented_journalism.pdf] [Consultado el: 20-04-2018].

Marconi, F (2017): "Report: How artificial intelligence will impact journalism”. Associated Press.[Disponible en: https:// insights.ap.org/industry-trends/report-how-artificial-intelligence-will-impact-journalism ] [Consultado el: 15/03/2018]

Martinez Sánchez, O:"Periodismo en la era digital:Los medios informativos, entre reacomodos, crisis y oportunidades". Revista Mexicana de Comunicación, Vol. 25, n.131, pp. 44-47. 
Mazlish, B (1995): “The man-machine and Artificial Intelligence”. Standford Electronic Humanities Review, n.4, pp: 21-45.

Minsky, M. (1986): La sociedad de la mente: de la inteligencia humana a la luz de la inteligencia artificial. Ediciones Galápago. Minsky, Marvin (1994): ¿Serán los robots quienes hereden la tierra?. Investigación y Ciencia, pp. 86-92.

Minsky, M. (2010): La máquina de las emociones: Sentido común, inteligencia artificial y el futuro de la mente humana. Barcelona: Random House Mondadori.

Moravec, Hans (1993): El hombre mecánico. El futuro de la robótica y la inteligencia humana. Barcelona: Salvat.

Newman, N. (2018): Journalism, Media, and Technology Trends and Predictions. Reuters Institute. [Disponible en: https:// reutersinstitute.politics.ox.ac.uk/sites/default/files/2018-01/RISJ\%20Trends\%20and\%20Predictions\%202018\%20NN.pdf ] [Consultado el: 01/04/2018].

Ornstein, Robert (1994): La evolución de la Conciencia: los límites del pensamiento racional. Salamandra.

Papadimitriou, A: The Future of Communication: Artificial Intelligence and Social Networks. Media \& Communication Studies. Mälmo University. [Disponible en:https://muep.mau.se/bitstream/handle/2043/21302/The\%20Future\%20of\%20 Communication.pdf?sequence=2 ] [Consultado el: 02/02/2018].

Pazos, J(2010): “Augusta Ada Byron, Condesa Lovelace, y su (in)merecida fama”. Manual formativo de ACTA, n.55, pp: 93-111 Ruiz Olabuénaga, J.L., Aristegui, I. y Melgosa, L. (2002): Cómo elaborar un proyecto de investigación social. Cuadernos monográficos del ICE. Bilbao: Universidad de Deusto.

Sánchez-Martín F.M., Jiménez Schlegl P., Millán Rodríguez F., Salvador-Bayarri J., Monllau Font V., Palou Redorta J., Villavicencio Mavrich H. (2007): “Historia de la robótica: de Arquitas de Tarento al Robot da Vinci”. Actas Urol Esp. vol.31 n.3.

Sáez Vacas, Fernando (1981). "El crepúsculo de cierta clase de Robots (una perspectiva histórico - científica de la robótica)”. Bit. Boletín informativo de telecomunicación, n. 19, pp. 34-41.

Saiz Lorca, D (2002): “R.U.R. de Capek: casi un siglo de robots”. Estadística Complutense, n.2 ,pp.211-218.

Sirvent, J. (2017). YoTubeTrabajo: Transformación Digital; susto o muerte. Jornadas Yo, Robot: puestos de trabajo que van a desaparecer. Madrid: ESIC.

Spielberg, S. (2001): AI: Artificial Intelligence. Estados Unidos: Warner Bros.

Terrén, E (2000): Desarrollo e implicaciones de la Revolución industrial. Ciencia, tecnología y sociedad. pp. 57-69.

Salazar García, I (2003): Internet como fuente de información: análisis de la red profunda y su utilidad periodística (Tesis doctoral). Universidad CEU San Pablo. Madrid.

Valencia Giraldo, A. (2004):De la técnica a la modernidad: construcciones técnicas, ciencia, tecnologían y modernidad. Colombia: Editorial Universidad de Antioquía, pp-228.

Ventura, D (2018): Amber Case: "Es absurdo tener miedo a la revolución de los robots, puesto que ya ha ocurrido". Huffingtonpost. [Disponible en: https://www.huffingtonpost.es/2018/01/05/amber-case-es-absurdo-tener-miedo-a-la-revolucion-de-los-robots-puesto-que-ya-ha-ocurrido_a_23297858/] [Consultado el 06/01/2018]. 
